\title{
Recombination and Loss Mechanisms in GaNAsP/GaP QW Lasers
}

\author{
N. Hossain ${ }^{1}$, J. Chamings ${ }^{1}$, S. R. Jin ${ }^{1}$, S. J. Sweeney ${ }^{1}$, S. Liebich ${ }^{2}$, S. Reinhard ${ }^{2}$, K. Volz ${ }^{2}$, B. Kunert ${ }^{3}$ and W. Stolz. ${ }^{2}$ \\ ${ }^{1}$ Advanced Technology Institute, University of Surrey, Guildford, Surrey GU2 7XH, UK. \\ ${ }^{2}$ Material Sciences Center and Faculty of Physics, Philipps-University, 35032 Marburg, Germany. \\ ${ }^{3} \mathrm{NAsP}$ III/V GmbH, Am Knechtacker 19, 35041 Marburg, Germany. \\ n.hossain@surrey.ac.uk
}

\begin{abstract}
In this paper the authors present a comprehensive study of the threshold current and its temperature dependence in novel direct band-gap $\mathrm{Ga}(\mathrm{NAsP}) / \mathrm{GaP}$ QW lasers which provide a potential route to lattice matched monolithic integration of long term stable semiconductor lasers on silicon. It is found that near room temperature, the threshold current is dominated by nonradiative recombination accounting for $\sim 87 \%$ of the total threshold current density. A strong increase in threshold current with hydrostatic pressure implies that a carrier leakage path is the dominant carrier recombination mechanism.
\end{abstract}

\section{INTRODUCTION}

Silicon photonics has been an active research area in recent years, with ultimate goal of this rapidly emerging field being the integration of individual photonic devices [1][8]. A commercial solution for the monolithic integration of long term stable laser diodes has not been achieved yet, which is the key device component to finally realize the optoelectronic integrated circuits (OEICs) and to fully profit from the concept of silicon photonics [9]. Large lattice mismatch between the conventional III/V laser materials like GaAs or InP to silicon leads unavoidably to the formation of high densities of threading dislocations in the integrated III/V layer during the epitaxial deposition of laser material on silicon substrates, which meant any long-term lasing operation of corresponding device structure. However, it has been shown that $\mathrm{GaP}$ can be grown without dislocations on silicon due to the relatively small difference in lattice constant $[<0.4 \%$ at room temperature] [10]. GaP is itself an indirect band gap semiconductor, but a $\mathrm{Ga}(\mathrm{NAsP})$ alloy with high As fractions and dilute $\mathrm{N}$ fractions (of $\sim 4 \%$ ) can form a direct band gap material approximately lattice matched to $\mathrm{GaP}$ and silicon [11]. Hence the realization of a GaP-based direct band gap semiconductor laser material on a silicon substrate can provide a realistic route toward monolithic laser sources for silicon-based OEICs. In this work, we investigate the temperature dependence and processes that limit device performance of novel direct band-gap $\mathrm{Ga}(\mathrm{NAsP}) / \mathrm{GaP}$ quantum well (QW) lasers. Using low temperature and high pressure techniques we have investigated the degree to which different carrier recombination processes govern laser behavior towards the optimization of lasers design parameters to improve their performance.

\section{LASER STRUCTURE}

The devices in this study were grown by metal organic vapor phase epitaxy (MOVPE) on a GaP substrate. They consist of a single $6 \mathrm{~nm} \quad \mathrm{Ga}\left(\mathrm{N}_{0.04} \mathrm{As}_{0.89} \mathrm{P}_{0.07}\right) \quad 2.5 \%$ compressively strained quantum well within two undoped $150 \mathrm{~nm} \mathrm{GaP}$ barrier/separate confinement layers. The GaP barrier layers are grown at $575{ }^{\circ} \mathrm{C}$ (sample A) and $675{ }^{\circ} \mathrm{C}$ (sample B). Optical confinement is provided by $1.8 \mu \mathrm{m}$ thick $\mathrm{Al}_{0.23} \mathrm{Ga}_{0.77} \mathrm{P}$ cladding layers doped $\mathrm{p}\left(\mathrm{Zn}: 7 \times 10^{18} \mathrm{~cm}^{-3}\right)$ and $\mathrm{n}\left(\mathrm{Te}: 2 \times 10^{18} \mathrm{~cm}^{-3}\right)$ forming the diode junction. The device stripes (50 and $100 \mu \mathrm{m}$ wide) were defined using regular masks and standard contacts were formed by deposition. The devices were measured as-cleaved with cavity length of $1000 \mu \mathrm{m}$.

\section{EXPERIMENTS}

Temperature dependence measurements over the range of 60-280K were performed with a standard closed cycle cryostat set-up. Hydrostatic pressure measurements over the range $0-8.5 \mathrm{kbar}$ were performed using a gas compressor system. Carrier recombination mechanisms are influenced by the band structure. Conventional methods to change the band structure to investigate the recombination mechanisms, involve growing a series of devices with a range of active region alloy compositions. The high costs involved with growing a lot of wafers, the difficulty in keeping doping densities and layer thicknesses identical mean this method can be unreliable. By using high hydrostatic pressure to reversibly alter the band-structure of one device, the recombination mechanisms can be quickly and easily resolved. The application of high hydrostatic pressure causes an increase in the direct band gap, mimicking the effect of alloying thereby allowing one to investigate wavelength dependent properties of semiconductor lasers. Furthermore, due to the different pressure coefficients of the layers it can also be used to alter the alignment between direct $(\Gamma)$ and indirect $(\mathrm{X}, \mathrm{L})$ bands and to alter the band offsets between layers of differing composition [12]. In order to quantify the different carrier recombination processes, we also measured the pure spontaneous emission from the lasers. Since the 
light emitted from the facets is strongly influenced by gain/loss as the optical field propagates along the cavity, we instead measure the light emitted in a perpendicular direction. To do this we milled a window in the n-side of the devices using a focused ion beam and aligned an optical fibre with the window to collect the spontaneous emission where the coupling efficiency is kept constant. This experiment was performed as a function of temperature. Further details of this technique are described elsewhere [13].

\section{TEMPERATURE DEPENDENCE}

The threshold current density $\left(J_{t h}\right)$ of device B (device A) is $\sim 3.2 \mathrm{kAcm}^{-2}\left(\sim 5.5 \mathrm{kAcm}^{-2}\right)$ with a lasing wavelength $\sim 968$ $\mathrm{nm}(\sim 954 \mathrm{~nm})$ at $280 \mathrm{~K}$, which is significantly improved than previously obtained values of $\sim 42 \mathrm{kAcm}^{-2}$ with a lasing wavelength of $\sim 942 \mathrm{~nm}$ at $278 \mathrm{~K}$ for $\mathrm{Ga}(\mathrm{NasP}) / \mathrm{GaP} \mathrm{QW}$ lasers [14]. However, our measured value of $J_{\text {th }}$ is larger than more established GaAs-based lasers, which operate at similar wavelengths for which $J_{\text {th }} / \mathrm{QW} \sim 100-200 \mathrm{Acm}^{-2}$ [15], [16], the cause of which became the focus of our investigations.

The threshold current of a semiconductor laser is the point at which the gain equals all of the losses in the device and above this current efficient stimulated emission dominates. It is therefore desirable to have a low (ideally zero) and temperature independent threshold current [17]. Figure-1a shows the normalized (at $\mathrm{T}=60 \mathrm{~K}$ ) temperature dependence of the $J_{t h}$. The characteristic temperature $\left(T_{0}\right)$ is the measure of temperature stability of threshold current and can be derived from the relation:

$$
\frac{1}{T_{0}}=\frac{d \ln \left(J_{t h}\right)}{d T}
$$

From figure-1a, we find that the $T_{0}$ (derived from equation-1) on device $\mathrm{B}$ (device $\mathrm{A})$ to be $\approx 108 \mathrm{~K}(107 \mathrm{~K})$ at $200 \mathrm{~K}$, which drops to $\approx 54 \mathrm{~K}(47 \mathrm{~K})$ at $280 \mathrm{~K}$, suggesting a thermally activated recombination process is becoming dominant at higher temperature in these devices. $T_{0}$ (at $280 \mathrm{~K}$ ) in device B is approximately the same as that of conventional InGaAsP/InP devices (typically $\sim 50-60 \mathrm{~K}$ near room temperature) and higher $(\sim 52 \mathrm{~K})$ than that for device $\mathrm{A}$ $(\sim 47 \mathrm{~K})$ over a temperature range of $200-280 \mathrm{~K}$, which is also consistent with the reduced $J_{t h}$ of device B than that of device A. Figure-1a also shows the temperature dependence of the radiative current density, $J_{\text {rad }}$, found from the pinning level of the integrated spontaneous emission has almost ideal QW linear temperature dependence (Figure-1b) leading to the conclusion that the loss mechanisms must increase superlinearly with increasing temperature. From the measured $J_{\text {th }}$ and $J_{\text {rad }}$ (in Figure-1a), for device B we estimate that the relative ratio of the radiative and non-radiative currents at $J_{t h}$ are $\sim 13 \%$ and $\sim 87 \%$, respectively, at $280 \mathrm{~K}$, compared with $\sim 9 \%$ and $\sim 91 \%$ for devices A at the same temperature. Thus, a non-radiative recombination process dominates $J_{t h}$ at room temperature. It can also be seen that in addition to having a lower absolute $J_{t h}$, device B has a slightly higher $J_{\text {rad }} / J_{t h}$ than in device A. To further probe this, high pressure techniques were utilized.


Fig. 1. (a) Normalised (at $T=60 \mathrm{~K}$ ) temperature dependence of $J_{t h}$. (b) Temperature dependence of $J_{r a d}$, has almost ideal QW linear temperature dependence.

\section{PRESSURE DEPENDENCE}

Light-current characteristics were measured at $80 \mathrm{~K}$ and $220 \mathrm{~K}$ for pressures $0-7 \mathrm{kbar}$, and corresponding threshold current $\left(I_{t h}\right)$ values were determined in the normal way. Lasing wavelengths $(\lambda)$ with pressure were measured at $1.1 I_{\text {th. }}$ Figure-2 shows the measured room temperature pressure dependence of $J_{t h}$. Also shown is the ideal expected variation of $J_{r a d} \alpha E_{g}^{2}[18]$, where $E_{g}$ is the bandgap (taken from $\left.E_{g}=h c / \lambda\right)$. $J_{t h}$ and $J_{\text {rad }}$ have been normalized to their respective values at atmospheric pressure. The pressure coefficient of the band gap for device B (device A) is $\sim 5.1$ $\mathrm{meV} / \mathrm{kbar}(\sim 4.2 \mathrm{meV} / \mathrm{kbar})$. It can be clearly seen that the threshold current increases with pressure much faster than $J_{\text {rad }}$, which confirms that the lasers are not operating in a radiatively dominated regime. The threshold current of device B (device A) is increased by $\sim 50 \%(\sim 31 \%)$ and $\sim 100 \%(56 \%)$ up to $7 \mathrm{kbar}$ at $80 \mathrm{~K}$ and $220 \mathrm{~K}$ respectively. The rapid increase in threshold current may indicate the presence of carrier leakage, where the leakage activation 
energy, $\Delta E$ (the energy required for carriers to escape from the $\mathrm{QW})$ reduces with increasing pressure. The pressure $(P)$ dependence of the leakage current, $J_{\text {leak }}$, can be written as [19]:

$$
J_{\text {leak }}(P)=J_{\text {leak }}(0) \exp \left(-\frac{d \Delta E}{d P} \frac{P}{k_{b} T}\right)
$$

where $k_{b}$ is the Boltzman's constant. If the leakage current competes against radiative recombination, and assuming negligible defect recombination, the pressure dependence of the $J_{t h}$ can be related to the relative changes of rate of radiative recombination and the rate of carrier leakage such as:

$$
\frac{J_{t h}(P)}{J_{\text {th }}(0)}=k_{\text {rad }} \frac{J_{\text {rad }}(P)}{J_{\text {rad }}(0)}+\left(1-k_{\text {rad }}\right) \frac{J_{\text {leak }}(P)}{J_{\text {leak }}(0)}
$$

where $k_{\text {rad }}=J_{\text {rad }} / J_{t h}$, the portion of carriers undergoing radiative recombination as opposed to carrier leakage.



Fig. 2. Pressure dependence of threshold current density. Also shown is the ideal expected variation of radiative current with pressure

From the ratio of $J_{r a d} / J_{t h}$ in figure-2a, the minimum fraction of leakage in the device B (device A) is $\sim 9 \%$ $(\sim 11 \%)$ and $\sim 87 \%(\sim 91 \%)$ at $80 \mathrm{~K}$ and $220 \mathrm{~K}$ respectively. We can use equations- 2 and 3 to calculate the rate of change of the leakage activation energy $(d \Delta E / d P)$ with pressure. The increase in threshold current with pressure is equated to the leakage current and then fitted to equation-2 to find $d \Delta E / d P$. We find that $d \Delta E / d P$ for device B (device A) is $-0.47(-0.3) \mathrm{meV} / \mathrm{kbar}$ and $-2.5(-1.6) \mathrm{meV} / \mathrm{kbar}$ at $80 \mathrm{~K}$ and $220 \mathrm{~K}$ respectively. This may indicated that the leakage path involves defect states in these devices. The lower rate of increase in threshold current with pressure in device A compared to device B may indicate the stronger presence of a leakage path which has only weak dependence on pressure, such as localized defects in or at the QW interface. A further quantitative study of radiative, leakage and defect related recombination processes in these devices is still in progress.

\section{CONCLUSIONS}

From our studies of $\mathrm{Ga}(\mathrm{NAsP}) / \mathrm{GaP}$ QW lasers, we find that near room temperature the threshold current is dominated by nonradiative recombination. The nonradiative process is also responsible for the poor temperature sensitivity of the devices resulting in low $T_{0}$ values near room temperature. From our pressure dependence measurements, we observe that the threshold current increases with pressure, consistent with the carrier leakage path which may involves localized defect states in these devices. If these leakage paths can be reduced, the growth of a direct band-gap material on GaP and eventually silicon substrate may lead to fully lattice matched silicon based OEICs operating at room temperature.

\section{REFERENCES}

[1] M. Paniccia, "Integrating silicon photonics," Nature Photonics UK, Vol. 4, pp. 498-499, 2010.

[2] T. Okumura, T. Maruyama, H. Yonezawa, N. Nishiyama, and S. Arai, "Injection-Type GaInAsP-InP-Si Distributed-Feedback Laser Directly Bonded on Silicon-on-Insulator Substrate," IEEE Photon. Technol. Lett. USA, Vol. 21, pp. 283-285, 2009.

[3] B. R. Koch, A. W. Fang, E. Lively, R. Jones, O. Cohen, D. J. Blumenthal, and J. E. Bowers, "Mode locked and distributed feedback silicon evanescent lasers," Laser \& Photon. Rev. Germany, Vol. 3, pp. 355-369, 2009.

[4] L. Chen, and M. Lipson, "Ultra-low capacitance and high speed germanium photodetectors on silicon," Opt. Express USA, Vol. 17, pp. 7901-7906, 2009.

[5] L. Chen, K. Preston, S. Manipatruni, and M. Lipson, "Integrated GHz silicon photonic interconnect with micrometer-scale modulators and detectors," Opt. Express USA, Vol. 17, pp. 15248-15256, 2009.

[6] G. T. reed, G. Mashanovich, F. Y. Gardes, and D. J. Thomson, "Silicon optical modulators," Nature Photonics UK, Vol. 4, pp. 518-527, 2010.

[7] C. R. Doerr, L. Chen, M. S. Rasras, Y.-K. Chen, J. S. Weiner, and M. P. Earnshaw, "Diplexer With Integrated Filters and Photodetector in Ge-Si using $\Gamma-\mathrm{X}$ and $\Gamma-\mathrm{M}$ Directions in a Grating Coupler," IEEE Photon. Technol. Lett. USA, Vol. 21, pp. 1698-1700, 2009.

[8] T. E. Kazior, J. R. Laroche, D. Lubyshev, J. M. Fastenau, W. K. Liu, M. Urteaga, W. Ha, J. Bergman, M. J. Choe, M. T. Bulsara, E. A. Fitzgerald, D. Smith, D. Clark, R. Thompson, C. Drazek, N. Daval, L. Benaissa, and E. Augendre, "Progress and challenges in the direct monolithic integration of III-V devices and Si CMOS on silicon substrates," Proc. IEEE Int. Conf. IPRM USA, pp. 100-104, 2009.

[9] B. Kunert, S. Liebich, S. Zinnkann, I. Nemeth, R. Fritz, K. Volz, W. Stolz, C. Lange, N. S. Koester, D. J. Franzbach, S. Chatterjee, W. W. Ruhle, N. C. Gerhardt, N. Koukourakis, and M. Hofmann, "Laser operation of the III/V compound material Ga(NAsP) grown lattice matched on (001) Si substrate," Proc.67th Device Research Conference, USA, pp. 213-214, 2009.

[10] H. Yonezu, "Control of structural defects in group III-V-N alloys grown on Si," Semicond. Sci. Technol. UK, Vol. 17, pp. 762-768, 2002.

[11] B. Kunert, K. Volz, J. Koch, and W. Stolz, "Direct-band-gap $\mathrm{Ga}(\mathrm{NAsP}) / \mathrm{GaP}-$ material system pseudomorphically grown on $\mathrm{GaP}$ substrate,"Appl. Phys. Lett. USA, Vol. 88, pp. 182108-182110, 2006.

[12] K. Hild, S. J. Sweeney, I. P. Marko, S. R. Jin, S. R. Johnson, S. A. Chaparro, S.-Q Yu, and Y-H. Zhang, "Temperature and pressure dependence of carrier recombination process in $\mathrm{GaAsSb} / \mathrm{GaAs}$ quantum well lasers," Physics Status Solidi B Germany, Vol. 244, pp. 197-202 2007.

[13] S. J. Sweeney, A. F. Philips, A. R. Adams, E. P. O'Reilly, and P. J. A. Thijs, "The effect of temperature dependent process on the performance of $1.5-\mu \mathrm{m}$ compressively strained $\operatorname{InGaAs}(\mathrm{P}) \mathrm{MQW}$ semiconductor diode lsers," IEEE Photonics Technol. Lett. USA, Vol. 10, pp. 10761078, 1998.

[14] B. Kunert, A. Klehr, S. Reinhard, K. Volz and W. Stolz, "Near room temperature electrical injection lasing for dilute nitride $\mathrm{Ga}(\mathrm{NAsP}) / \mathrm{GaP}$ 
quantum-well structures grown by metal organic vapour phase epitaxy," Elec. Lett. UK, Vol. 42, pp. 601-603, 2006.

[15] J. Chamings, S. J. Sweeney, A. R. Adams, B. Kunert, K. Volz, W. Stolz, "Temperature dependence and physical properties of $\mathrm{Ga}(\mathrm{NAsP}) / \mathrm{GaP}$ semiconductor lasers," Appl. Phys. Lett. USA, Vol. 93, pp. 101108101111,2011

[16] G. Adolfsson, S. M. Wang, M. Sadeghi, and A. Larsson, "Highperformance long-wavelength InGaAs/GaAs multiple quantum-well lasers grown by molecular beam epitaxy," Electron. Lett UK, Vol. 43, pp. 454-456, 2007.
[17] N. Hossain, S. R. Jin, S. J. Sweeney, S. Yu, S. R. Johnson, D. Ding, and Y. H. Zhang, "Improved Performance of GaAsSb/GaAs SQW Lasers," Proceedings of SPIE USA, Vol. 7616, pp. 1-5, 2010.

[18] A.R. Adams, M. Silver and J. Allam, "Semiconductors and Semimetals," Semiconductor Optoelectronic Devices UK, Vol. 55, pp. 301-352, 1998.

[19] S. R. Jin, S. J. Sweeney, S. Tomic, A. R. Adams, and H. Riechert, "High-Pressure Studies of Recombination Mechanisms in $1.3 \mu \mathrm{m}$ GaInNAs Quantum-Well Lasers," IEEE J. of Select. Topics in Quantum Electron. USA, Vol. 9, pp. 1196-1201, October 2003. 